\title{
Undernutrition during the first trimester of pregnancy does not influence fetal growth, but may affect the development of the circulatory system in cattle
}

\author{
F. Mossa ${ }^{1}$, T. T. Hildebrandt ${ }^{2}$, M. Mihm $^{3}$, D. Kenny ${ }^{1}$, S. T. Butler ${ }^{4}$, T. Fair ${ }^{1}$, \\ P. Lonergan ${ }^{1}$ and A. C. O. Evans ${ }^{1}$ \\ ${ }^{1}$ School of Agriculture Food Science and Veterinary Medicine, University College Dublin, Belfield, Dublin 4, Republic of \\ Ireland, ${ }^{2}$ Leibniz Institute for Zoo and Wildlife Research, PF 601 103, 10252 Berlin, Germany, ${ }^{3}$ Division of Cell Sciences, \\ ICM, Faculty of Veterinary Medicine, University of Glasgow, Glasgow, UK and ${ }^{4}$ Teagasc, Moorepark Dairy Research \\ Centre, Fermoy, Co. Cork, Republic of Ireland
}

Empirical and scientific studies in a number of species have shown that maternal nutritional status during the early stages of pregnancy affects fertilisation, implantation, placental development, foetal organogenesis and growth ${ }^{(1)}$. However, in cattle more emphasis has been placed on the study of nutrition during late pregnancy, when exponential fetal growth occurs ${ }^{(2)}$. The aim of the present work was to study the effects of nutritional restriction from $11 \mathrm{~d}$ before conception to the end of the first trimester of pregnancy (day 110) on fetal development in cattle.

Cross-bred beef heifers ( $n$ 60) were randomly assigned to one of two nutritional treatments: control $(\mathrm{C} ; n$ 25) or restricted (R; $n$ 35) and were fed at 1.2 or $0.6 \times$ maintenance requirements respectively. The heifers were individually fed, starting $11 \mathrm{~d}$ before artificial insemination. Oestrous cycles were synchronized and the heifers that responded to the treatment $(\mathrm{C}, n 22 ; \mathrm{R}, n$ 28) were impregnated using gender-sorted semen from a single sire to ensure heifer calves. Pregnancy was determined on day 28 using ultrasonography. On day 94 of pregnancy fetal development was monitored through transrectal ultrasonography in a subset of animals using a Voluson ${ }^{\circledR} \mathrm{I}(\mathrm{GE}$ Healthcare, Munich, Germany) with a $4-8 \mathrm{MHz}$ probe. Two-dimensional images were digitally stored and subsequently analysed using a GE 4DView (GE Healthcare) and Sonoview software (Sonometrics Corp., London, Ont., Canada). The following fetal structures were measured: length of long bones (humerus, radius, femur and tibia), biparietal distance (diameter of the head), eyeball and eye lens maximal diameter, umbilical cord (width at base, diameters in a cross-section) and associated blood vessels, maximal diameter of the thorax and of the cotyledon. The maximal diameter of the left ventricle at the end of diastole and systole and the diameter of the aorta were measured in a four-chamber view. Significant difference in pregnancy rate was determined by $\chi^{2}$ test. All measurements are expressed as mean with their standard errors $(\mathrm{mm})$ and independent $t$ tests were used to determine whether group means differed significantly $(P<0.05)$.

There was no difference in pregnancy rate on day $28(\mathrm{C}, 59 \%, n 13 ; \mathrm{R}, 53 \%, n 15)$. In addition, on day 94 the length of fetal long bones (femur; C, 14.4 (SE 0.49), $n$ 9; R, 14.5 (SE 0.44), $n$ 7), thorax maximal diameter (C, 37.2 (SE 1.04), $n$ 11; R, 37.5 (SE 2.81), $n$ 7) and other skeletal structures measured did not differ. Aorta diameter was larger $(P<0.05)$ in the $\mathrm{R}$ group $(3.2(\mathrm{sE} 0.17), n 7)$ compared with the $\mathrm{C}$ group (2.6 (SE 0.11), $n$ 10), while no significant difference was found in the width of the umbilical cord (C, 11.8 (SE 0.51$), n 8 ; \mathrm{R}, 11.4$ (SE 0.66), $n$ 9), in the maximal diameter of the cotyledons (C, 24.3 (SE 2.13), $n$ 8, R, 24.7 (SE 2.16), $n$ 4) or in the maximal diameter of the left ventricle at the end of diastole (C, 6.4 (SE 0.41), $n$ 8; R, 6.1 (SE 0.31), $n$ 4) or systole (C, 3.9 (SE 0.49), $n$ 6; R, 4.0 (SE 0.47), $n$ 3).

It is concluded that maternal nutritional restriction during the first trimester of pregnancy does not impair fetal skeletal growth, but may affect the development of the circulatory system, as seen by an increase in the diameter of the aorta. Whether the detected difference has long-term consequences and is permanent after birth is under investigation.

1. Rhind SM (2004) Anim Reprod Sci 82-3, 169-181.

2. Roche JF (2006) Anim Reprod Sci 96, 282-296. 